\title{
Allogeneic hematopoietic stem cell transplantation should be in preference to conventional chemotherapy as post-remission treatment for adults with lymphoblastic lymphoma
}

\author{
Luxin Yang ${ }^{1} \cdot$ Yamin Tan ${ }^{1} \cdot$ Jimin Shi ${ }^{1} \cdot$ Yanmin Zhao ${ }^{1}$ Yuanyuan Zhu ${ }^{1} \cdot$ Yongxian Hu${ }^{1} \cdot$ Wenjue Pan ${ }^{1} \cdot$ Yishan ${ }^{1}$. \\ Jingsong $\mathrm{He}^{1} \cdot$ Weiyan Zheng ${ }^{1} \cdot$ Jie Sun ${ }^{1} \cdot \mathrm{Zhen} \mathrm{Cai}^{1} \cdot \mathrm{He} \mathrm{Huang}^{1} \cdot \mathrm{Yi} \mathrm{Luo}^{1}$
}

Received: 2 January 2018 / Revised: 8 March 2018 / Accepted: 19 March 2018 / Published online: 30 April 2018

(c) The Author(s) 2018. This article is published with open access

Lymphoblastic lymphoma (LBL) is a rare and aggressive disease, accounting for less than $2 \%$ of non-Hodgkin's lymphoma (NHL) [1, 2]. Unlike lymphoblastic leukemia (ALL), LBL occurs mainly in young males and is clinically characterized by mass in lymph nodes and extranodal organs with no or minimal evidence of bone marrow involvement $(<20 \%)$. The response rates have been greatly improved with intensive chemotherapy protocols used for NHL or ALL. However, because of high risk of relapse [3], long-term overall survival (OS) of LBL remains unsatisfactory, with a 5-year OS of $32 \%$ and event-free survival of $22 \%$ using NHL protocols [4]. It has been reported that allogeneic stem cell transplantation (allo-SCT) achieved lower relapse rates and improved survival in patients with recurrent or refractory disease and those at high risk of resistance or relapse $[4,5]$. However, the timing of alloSCT and which patients may benefit from the treatment are under debate.

We retrospectively analyzed 57 consecutive patients with LBL diagnosed between January 2006 and December 2016 in our institution. Most patients were young males, with $88 \%$ of patients having a T-cell immunophenotype. Mediastinal mass was the most common clinical presentation $(53 \%, n=30)$. Medullary involvement was observed in 29 $(51 \%)$ patients, while pleural or pericardial involvement

Electronic supplementary material The online version of this article (https://doi.org/10.1038/s41409-018-0184-7) contains supplementary material, which is available to authorized users.

Yi Luo

luoyijr@zju.edu.cn

1 Bone Marrow Transplantation Center, The First Affiliated Hospital, School of Medicine, Zhejiang University, Hangzhou, People's Republic of China was noted in $11(19 \%)$ patients. As a result, $88 \%$ of the patients presented with an advanced disseminated disease (III/IV stage). Nearly half of the patients had highintermediate and high international prognosis index (IPI) $(44 \%, n=25)$. More details about clinical characteristics were listed in Supplementary Table 1. Induction chemotherapies mainly consisted of the hyper-CVAD regimen $(54 \%, n=31)$ and the CHOP and CHOP-based regimens $(31 \%, n=18)$ (Supplementary Table 2). After four courses of induction chemotherapy, 43 patients responded, with an overall response rate of $75 \%$ (complete remission (CR): 54\%; partial remission (PR): 21\%). Local radiotherapy was provided to patients with residual tumor in the mediastinum $(n=4)$ or neck $(n=1)$ after induction chemotherapy with a dose ranging from 20 to 40 Gray. All the 43 responders were completely ambulatory (Eastern Cooperative Oncology Group performance status 0 or 1) after induction chemotherapy. The decision of whether performing a transplantation or chemotherapy after remission was made according to the availability of suitable donor and patient's willingness. Given the aggressiveness of LBL and GVL effect associated with allogeneic grafts, allogeneic hematopoietic stem cell transplantation (allo-HSCT) was considered prior to auto-HSCT in our center. For patients who aimed to allo-HSCT, a matched sibling donor (MSD) was first preferred, then a matched unrelated donor (MUD), a haploidentical-related donor (HRD) was selected when neither MSD or MUD was available. Conditioning regimen and graft-versus-host disease (GVHD) prophylaxis were carried out as described previously [6]. Responders who did not have a suitable donor or the will to undergo transplantation continued with the effective chemotherapy they had for induction. Those who failed to achieve at least a PR received salvage intensified chemotherapy and were excluded from further analysis. OS and progression-free survival (PFS) were estimated using the Kaplan-Meier 
Table 1 Characteristics of patients in different post-remission therapy groups

\begin{tabular}{llll}
\hline Variable & Total & Allo- & Chemotherapy $p$ \\
& & HSCT \\
& group
\end{tabular}

\begin{tabular}{lllll}
\hline$N$ & 39 & 17 & 22 & \\
$\begin{array}{l}\text { Gender (\%) } \\
\quad \text { Male }\end{array}$ & $27(69)$ & $13(76)$ & $14(64)$ & 0.494 \\
$\quad$ Female & $12(31)$ & $4(24)$ & $8(36)$ & \\
$\begin{array}{l}\text { Age years median } \\
\text { (range) }\end{array}$ & $26(15-61)$ & $\begin{array}{l}26 \\
(15-52)\end{array}$ & $28(15-61)$ & 0.821 \\
Immunophenotye & & & & 1.000
\end{tabular}

$(\%)$

$\mathrm{T} \quad 34(87) \quad 15(88) \quad 19(86)$

B $\quad 5(13) \quad 2(12) \quad 3(14)$

Ann Arbor stage (\%)

$\begin{array}{llll}\text { I } & 2(5) & 1(6) & 1(5) \\ \text { II } & 1(3) & - & 1(5) \\ \text { III } & 7(18) & 3(18) & 4(20) \\ \text { IV } & 27(69) & 13(76) & 14(70) \\ \text { N/A } & 2(5) & - & 2(10)\end{array}$

B symptoms (\%)

Absent
Present
N/A

IPI Index $(\%)$

0 or 1
2
3
4 or 5
N/A

\section{ECOG-PS (\%)}

0
1
2
3

Serum LDH level > normal (\%)

Yes
No
N/A

Medullary

involvement (\%)

$\begin{array}{llll}\text { Yes } & 22(56) & 9(53) & 13(59) \\ \text { No } & 16(41) & 8(47) & 8(36) \\ \text { N/A } & 1(3) & - & 1(5)\end{array}$

Medullary involvement (\%)

$\begin{array}{llll}\leq 5 \% & 29(74) & 13(76) & 16(73) \\ >5 \% & 8(21) & 3(18) & 5(23)\end{array}$

0.449

0.647

$\begin{array}{lll}23(59) & 9(53) & 14(63) \\ 13(33) & 7(41) & 6(27) \\ 3(8) & 1(6) & 2(10)\end{array}$

Table 1 (continued)

\begin{tabular}{lllll}
\hline Variable & Total & $\begin{array}{l}\text { Allo- } \\
\text { HSCT } \\
\text { group }\end{array}$ & $\begin{array}{l}\text { Chemotherapy } \\
\text { group }\end{array}$ & $p$ \\
\hline N/A & $2(5)$ & $1(6)$ & $1(4)$ & 0.367 \\
$\begin{array}{l}\text { Mediastinal } \\
\text { involvement (\%) }\end{array}$ & & & & \\
$\quad$ Yes & $21(54)$ & $8(47)$ & $13(59)$ & \\
No & $17(44)$ & $9(53)$ & $8(36)$ & $1(5)$ \\
N/A & $1(2)$ & - & &
\end{tabular}

Pleural/pericardial effusion (\%)

$\begin{array}{llll}\text { Yes } & 8(21) & 4(24) & 4(18) \\ \text { No } & 30(77) & 13(76) & 17(77) \\ \text { N/A } & 1(2) & - & 1(5)\end{array}$

Number of extranodal sites (\%)

$\begin{array}{llll}0 & 7(18) & 3(18) & 4(18) \\ 1 & 17(44) & 6(35) & 11(50) \\ \geq 2 & 13(33) & 8(47) & 5(23) \\ \text { N/A } & 2(5) & - & 2(9)\end{array}$

Induction

chemotherapy

\begin{tabular}{lllll}
$\begin{array}{l}\text { Hyper-CVAD A/ } \\
\text { B }\end{array}$ & 10(59) & $13(59)$ & \\
$\quad \begin{array}{lll}\text { CHOP/CHOP } \\
\text { like }\end{array}$ & $12(31)$ & $4(24)$ & $8(36)$ & \\
$\quad$ Others & $4(10)$ & $3(17)$ & $1(5)$ & 0.752 \\
Disease status & & & & \\
$\quad$ CR & $28(72)$ & $13(76)$ & $15(68)$ & 1.000 \\
PR & $11(28)$ & $4(24)$ & $7(32)$ & \\
Radiation therapy & & & & \\
Yes & $4(10)$ & $2(12)$ & $2(10)$ & \\
$\quad$ No & $35(90)$ & $15(88)$ & $18(90)$ & \\
Donor type & & & & \\
MSD & - & $7(40)$ & - \\
MUD & - & $5(30)$ & - \\
HRD & - & $5(30)$ & - \\
\hline
\end{tabular}

ECOG-PS Eastern Cooperative Oncology Group Performance Status, $L D H$ lactate dehydrogenase, IPI international prognosis index, N/A not available, $C R$ complete remission, $P R$ partial remission, Hyper-CVAD $A$ cyclophosphamide, vincristine, doxorubicin, and dexamethasone, Hyper-CVAD B methotrexate, cytarabine, $C H O P$ cyclophosphamide, doxorubicin, vincristine, prednisolone, $M S D$ matched sibling donor, $M U D$ matched unrelated donor, $H R D$ haploidentical-related donor

method. The Cox proportional hazards regression model was used for multivariate analysis to compare factors with a $p$-value $\leq 0.2$ in the univariate analysis. Cumulative incidences of relapse and non-relapse mortality (NRM) were calculated by the competing risk method [7]. A two-sided $p$-value $<0.05$ was considered statistically significant. 
Fig. 1 Overall survival (a), progression-free survival (b), cumulative incidence of relapse (c), and non-relapse mortality (d) for patients in Allo-HSCT group and chemotherapy group. Allo-HSCT allogeneic hematopoietic stem cell transplantation
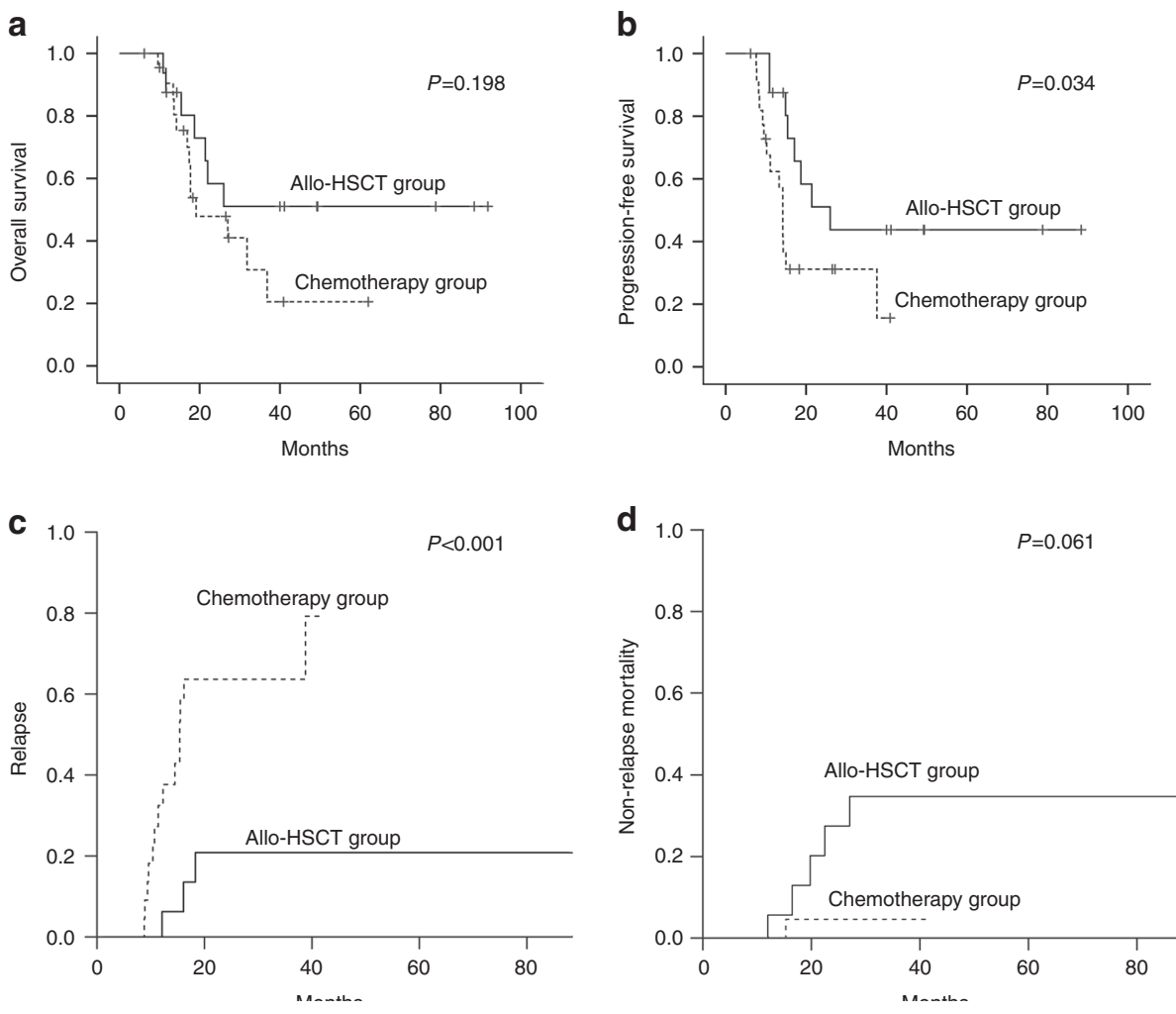

Twenty patients underwent first-line SCT (17 allo-HSCT and 3 auto-HSCT), while 22 patients continued with chemotherapy. The median interval from the completion of induction chemotherapy to allo-HSCT was 3.8 (range 1.0-8.4) months, one patient was excluded from the chemotherapy group because of the short remission duration of 3.6 months. The distribution of clinical characteristics was well balanced between the two groups (Table 1). The median cycles of chemotherapy offered in chemotherapy group was 7 (range 4-10 cycles). The maintenance therapy included oral 6-mercaptopurine and methotrexate for 2 years in three patients, whereas the other seven patients were observed without additional treatment. Summary of the treatments and responses are displayed in Supplementary Fig. 1. With a median follow-up of 27.2 (range 6.2-91.8) months for surviving patients, the 2-year OS and PFS for all responders were 56 and $35 \%$, respectively (Supplementary Fig. 2). The 2-year PFS was 51\% (95\% CI, $25-77 \%$ ) for the allo-HSCT group, comparing with $31 \%$ (95\% CI, $11-53 \%)$ for the chemotherapy group $(p=0.034)$ (Fig. 1a). Meanwhile, the corresponding figures for OS were $58 \%$ (95\% CI, 32-84\%) and 48\% (95\% CI, 25-71\%), respectively ( $p=0.198$ ) (Fig. 1b). The 2-year cumulative incidence of relapse and NRM for the two groups were $14 \%$ vs. $47 \%(p<0.001), 28 \%$ vs. $5 \%(p=0.061)$, respectively (Fig. 1c, d). The causes for death after allo-HSCT was infections $(n=3)$, disease progression $(n=2)$, chronic GVHD (cGVHD) $(n=1)$, and secondary graft failure $(n=$
1). A total of 13 deaths occurred in the chemotherapy group (relapsed/progressive disease $n=10$, infection $n=2$, osteofascial compartment syndrome $n=1$ ). Unfortunately, all the three patients who autografted relapsed and died. Of the 17 patients who underwent allo-HSCT, 16 patients engrafted successfully. Two patients developed grade-III acute GVHD (aGVHD) and two developed severe cGVHD.

In univariate analyses, more extranodal involvements $(p=0.01)$ and poor Eastern Cooperative Oncology Group performance status (ECOG-PS $>2$ ) at diagnosis were associated with worse OS $(p<0.001)$. ECOG-PS $>2$ at diagnosis $(p=0.002)$ and advanced Ann Arbor stage $(p=0.053)$ significantly affected PFS negatively. When entered into multivariate analysis of PFS, only baseline ECOG-PS $>2$ $(p=0.009$; HR: 8.158 ; 95\% CI: 1.6794-39.636) achieved statistical significance. Worse OS was predicted by ECOG-PS $>2 \quad(p=0.001 ; \quad$ HR: $75.496 ; \quad 95 \% \quad$ CI: 6.536-872.080) and radiotherapy ( $p=0.017$; HR: 4.725; 95\% CI: 1.325-16.850) (data not shown).

To the best of our knowledge, this is the largest study that compare allo-HSCT with conventional chemotherapy for unselected adults with LBL in first remission. The 2-year PFS was $51 \%$ in allo-HSCT group which was significantly superior to that of chemotherapy group $(p=$ 0.034). There was a tendency for longer OS in the allo-SCT group but it failed to reach statistical significance, on account of small number of patients and short follow-up. 
Noteworthily, allo-SCT group was associated with significantly less relapses and a comparable NRM. Allo-HSCT was given to unselected patients regardless of risk stratification and performance status and $24 \%$ of patients underwent transplant in PR, perhaps accounting for lower survival rates compared with that of historical studies [8-10]. Even better outcomes were achieved by intensive ALL-type chemotherapy regimen without consolidation transplant $[2,11]$. In the present study, the hyper-CVAD regimen showed a significantly higher $\mathrm{CR}$ rate than $\mathrm{CHOP}$ and CHOP-based regimen ( 68 vs. $39 \%, p=0.049$ ). Thomas et al. [11] reported that the 3-year PFS was $66 \%$ in 33 adults after the hyper-CVAD regimen. Also, a large prospective study reported a 2-year DFS of $72.4 \%$ with a pediatric-like ALL chemotherapy, while post-remission allo-SCT $(n=17)$ did not bring any survival advantages [2]. However, patients included in the studies mentioned above were less aggressive and the limited cases of allo-SCT were hardly to make definitive conclusions. Although the results of intensive ALL-type regimen are very encouraging, the efficiency of chemotherapy is greatly compromised due to the severe hematological toxicity in Asians [12]. As a result, patients would benefit more from the upfront use of alloSCT because of the shorten chemotherapy treatment duration and superior outcome brought with it.

The mediastinum is the most frequent site of presentation and recurrence, but the role of radiotherapy remains controversial. Our multivariate analysis suggested that radiotherapy associated with a statistically worse OS. Similarly, a large prospective study [13] found a better OS in the patients who did not received MedRad therapy (MRT) ( $p=0.07)$, comparing with the prophylaxis MRT cohort (36 Gray). Considering the increasing risks of secondary disease and inferior survival, radiotherapy should be provided with caution.

To date, reliable prognostic factors have not been identified in LBL. Coleman et al. [14] proposed a risk statification for LBL patients in 1986. High-risk patients (defined as Ann Arbor stage IV disease with bone marrow or central nervous system involvement or initial elevated lactate dehydrogenase concentration) had a 5-year relapse-free survival rate of $19 \%$ compared with $94 \%$ for the low-risk patients $(p=.0006)$. Despite that the Coleman system has been widely accepted, most further studies failed to identified these prognostic factors. Our risk factor analysis indicated poor ECOG-PS at diagnosis was an independent risk factor for both OS and PFS, which was in accordance with previous conclusions $[2,15]$. One possible explanation was that higher tumor burden resulted in poorer performance status. Recently, novel risk factors such as molecular marker (NOTCH/FBXW7/RAS/PTEN) [2] have emerged and are waiting to be evaluated.
Our previous data demonstrated that allo-HSCT from suitable HRDs achieved similar outcome to MUDs and improved the outcome of high-risk leukemia [6]. In our current study, five patients received haploidentical grafts and three of them were in continuous remission. In view of the immediate availability of a HRD and promising clinical outcome, haplo-HSCT seems to be an alternative option for LBL.

In conclusion, this study underlines the superiority of allo-HSCT over conventional chemotherapy in adults with LBL in their first remission. More studies are needed to identify potential long-term toxicities and life quality after allogeneic hemopoietic stem cell transplantation.

Acknowledgements The authors sincerely thank the participants for their help and willingness to participate in this study. This work was supported by the National Natural Science Foundation of China (81370644, YL).

\section{Compliance with ethical standards}

Conflict of interest The authors declare that they have no conflict of interest.

Open Access This article is licensed under a Creative Commons Attribution 4.0 International License, which permits use, sharing, adaptation, distribution and reproduction in any medium or format, as long as you give appropriate credit to the original author(s) and the source, provide a link to the Creative Commons license, and indicate if changes were made. The images or other third party material in this article are included in the article's Creative Commons license, unless indicated otherwise in a credit line to the material. If material is not included in the article's Creative Commons license and your intended use is not permitted by statutory regulation or exceeds the permitted use, you will need to obtain permission directly from the copyright holder. To view a copy of this license, visit http://creativecommons. org/licenses/by/4.0/.

\section{References}

1. Armitage JO, Weisenburger DD, Proj N-HLC. New approach to classifying non-Hodgkin's lymphomas: clinical features of the major histologic subtypes. J Clin Oncol. 1998;16:2780-95.

2. Lepretre S, Touzart A, Vermeulin T, Picquenot JM, TanguySchmidt A, Salles G, et al. Pediatric-like acute lymphoblastic leukemia therapy in adults with lymphoblastic lymphoma: the GRAALL-LYSA LL03 study. J Clin Oncol. 2016;34:572-80. https://doi.org/10.1200/JCO.2015.61.5385.

3. Hoelzer D, Gokbuget N, Digel W, Faak T, Kneba M, Reutzel R, et al. Outcome of adult patients with T-lymphoblastic lymphoma treated according to protocols for acute lymphoblastic leukemia. Blood. 2002;99:4379-85. https://doi.org/10.1182/blood-2002-010110.

4. Le Gouill S, Lepretre S, Briere J, Morel P, Bouabdallah R, Raffoux $\mathrm{E}$, et al. Adult lymphoblastic lymphoma: a retrospective analysis of 92 patients under 61 years included in the LNH87/93 trials. Leukemia. 2003;17:2220-4. https://doi.org/10.1038/sj.leu. 2403095.

5. Brammer JE, Khouri I, Marin D, Ledesma C, Rondon G, Ciurea $\mathrm{SO}$, et al. Stem cell transplantation outcomes in lymphoblastic 
lymphoma. Leuk Lymphoma. 2017;58:366-71. https://doi.org/10. 1080/10428194.2016.1193860.

6. Luo Y, Xiao HW, Lai XY, Shi JM, Tan YM, He JS, et al. T-cellreplete haploidentical HSCT with low-dose anti-T-lymphocyte globulin compared with matched sibling HSCT and unrelated HSCT. Blood. 2014;124:2735-43. https://doi.org/10.1182/blood2014-04-571570.

7. Scrucca L, Santucci A, Aversa F. Competing risk analysis using R: an easy guide for clinicians. Bone Marrow Transplant. 2007;40:381-7. https://doi.org/10.1038/sj.bmt.1705727.

8. Milpied N, Ifrah N, Kuentz M, Maraninchi D, Colombat P, Blaise $\mathrm{D}$, et al. Bone marrow transplantation for adult poor prognosis lymphoblastic lymphoma in first complete remission. Br J Haematol. 1989;73:82-87.

9. Levine JE, Harris RE, Loberiza FR Jr., Armitage JO, Vose JM, Van Besien $\mathrm{K}$, et al. A comparison of allogeneic and autologous bone marrow transplantation for lymphoblastic lymphoma. Blood. 2003;101:2476-82. https://doi.org/10.1182/blood-200205-1483.

10. Sweetenham JW, Santini G, Qian W, Guelfi M, Schmitz N, Simnett S, et al. High-dose therapy and autologous stem-cell transplantation versus conventional-dose consolidation/maintenance therapy as postremission therapy for adult patients with lymphoblastic lymphoma: results of a randomized trial of the European Group for Blood and Marrow Transplantation and the United Kingdom Lymphoma Group. J Clin Oncol. 2001;19:2927-36. https://doi.org/10.1200/JCO.2001.19.11.2927.

11. Thomas DA, O'Brien S, Cortes J, Giles FJ, Faderl S, Verstovsek $\mathrm{S}$, et al. Outcome with the hyper-CVAD regimens in lymphoblastic lymphoma. Blood. 2004;104:1624-30. https://doi.org/10. 1182/blood-2003-12-4428.

12. Jeong SH, Moon JH, Kim JS, Yang DH, Park Y, Cho SG, et al. Multicenter analysis of treatment outcomes in adult patients with lymphoblastic lymphoma who received hyper-CVAD induction followed by hematopoietic stem cell transplantation. Ann Hematol. 2015;94:617-25.

13. Gokbuget N, Wolf A, Stelljes M, Huttmann A, Buss EC, Viardot A et al. Favorable outcome in a large cohort of prospectively treated adult patients with T-lymphoblastic lymphoma (T-LBL) despite slowly evolving complete remission assessed by conventional radiography. Blood. 2014;124(21).

14. Coleman CN, Picozzi VJ, Cox RS, Mcwhirter K, Weiss LM, Cohen JR, et al. Treatment of lymphoblastic lymphoma in adults. J Clin Oncol. 1986;4:1628-37.

15. TVSVGK Tilak, Raina V, Kumar L, Sharma A, Sharma MC, Vishnubhatla $S$, et al. Superior vena cava syndrome and poor performance status at presentation affect survival in mediastinal Tlymphoblastic lymphoma-a single institute experience from India. Ann Hematol. 2013;92:917-23. https://doi.org/10.1007/s00277013-1727-z. 